\title{
Professional guidelines on Decisions Relating to Cardiopulmonary Resuscitation: introduction
}

The context in which the British Medical Association (BMA) first considered publishing specific guidelines on decisions about attempting cardiopulmonary resuscitation (CPR), in the early 1990s, needs to be remembered. At that time the subject was often seen as far too sensitive to be mentioned to patients. Many hospitals had no formal policy about how CPR decisions should be made, apart from an expectation that these were purely medical matters. Advance decision making about CPR, where it existed, appears to have been generally on an ad hoc basis by doctors. Patients, their relatives and the rest of the health care team were often unaware of it. After several cases in which nurses had been disciplined-unfairly in the eyes of some-for complying with their patients' known wishes about non-resuscitation in cases where no advance medical decision had been recorded, there was a demand for guidance.

The BMA was approached by nurses' representatives with a view to publishing some very basic advice. This advice, issued in 1993 jointly with the Royal College of Nursing, and in association with the Resuscitation Council, was under 1000 words. Whether this brevity reflects difficulties in achieving consensus or lower expectations about what was required is a matter for speculation. Throughout the 1990s the guidance was distributed widely and used as a basis for many hospitals' local policies. Recently, however, the demands on the BMA and other professional bodies for ethical advice have changed and grown. Discussion of sensitive decisions about potentially life-prolonging interventions is now de rigueur rather than taboo. Doctors no longer have the sole burden and privilege of deciding. It may also be that health professionals feel more uncertain, particularly on matters of law and ethics. When professional guidance is issued, there is considerable pressure from health workers, the public and the media to justify and explain it.

For example in 1993, the CPR guidelines identified three situations in which it was appropriate to consider a decision not to resuscitate a patient: where the patient's condition indicates that effective CPR is unlikely to be successful; where CPR is not in accord with the recorded, sustained wishes of the patient who is mentally competent, and where successful CPR is likely to be followed by a length and quality of life which would not be acceptable to the patient. By the time of the 2001 revision, exploration of these points alone occupies more paper than the whole of the previous document.

The concepts in the new version are no longer new for health professionals although some of the terminology may be. An important impetus for updating the guidelines was the Human Rights Act 1998 which requires health professionals to consider patients' rights as part of decision making. The principles that underpin the act-most significantly respect for human dignity and respect for legality-are not new, and the balancing of competing interests is familiar territory. But doctors are now required to think about their decisions using a new legal language that was formulated with gross abuses of human rights in mind. It is not immediately apparent how the right to be free from "torture or . . . inhuman or degrading treatment" (article 3) is relevant to decisions about whether to attempt CPR, but doctors must be able to show they have considered whether this right is affected by their decisions.

There was speculation before the act's implementation in October 2000 that decisions to withhold or withdraw potentially life-prolonging treatment would be challenged under the protection of the "right to life" (article 2). But legal advice to the BMA said it was improbable that the courts would turn this guarantee into a positive obligation to prolong life at all costs. The matter may be clarified by a legal case that is outstanding at the time of writing, in which a patient is seeking to challenge a "do not attempt resuscitation" order. If the outcome of the case has implications for the guidance, advice will be put on the BMA's website (www.bma.org.uk).

Wherever possible, the guidelines avoid what might be termed jargon, even where this is traditional terminology. Terms such as "quality of life", "futility" and even "best interests" can sound paternalistic and dismissive, and have become an often poorly understood shorthand for the real issues, with usage and precise definitions varying. 
For example some might describe attempting CPR as "futile" only if it cannot restart the patient's heart and lungs. Others would use the term with a wider meaning, to include also, where it can maintain the function of these organs but keeps the patient alive without awareness or in unmanageable pain and distress. Without a definition of "futility" it is not clear which is meant. The BMA believes that what is important is whether treatment can provide an overall benefit to the patient. Rather than thinking about whether a treatment is "futile", health professionals are asked to identify and weigh up the pros and cons for the particular patient. Considering where the balance lies is familiar to health professionals, and hopefully the "benefits and burdens" model in the guidelines is useful in a range of ethical decision making.

Producing the guidelines followed the usual BMA route: discussion in the medical ethics committee (which comprises elected representatives of the medical profession and appointed academics from disciplines including law, mora philosophy, ethics, social policy and theology) and consultation, in this case with other BMA committees, professional organisations and patient groups. A key message from the consultation was the vast range of variables that require consideration in individual cases, so that although the underlying ethical and legal principles might be the same, the way decisions are made; the practicalities of when and how to talk to patients; what type of written information to give to patients and people close to them; what to do if the clinician in charge is not available, and how to communicate decisions effectively are matters for local advice. The guidelines should be used as a framework within which to build policies to address the needs of the particular patient population.

A recommendation in the guidelines is that patients should be provided with written information about what CPR is and how decisions about it are made, and the BMA is working on a draft model information leaflet for patients and people close to them. British Medical Association members are concerned that people do not understand what CPR is, what it involves and how likely it is to be successful. A better level of understanding within the population should help when doctors come to talk to their patients about whether to attempt it in the event of cardiopulmonary arrest. The draft leaflet explains how decisions will be made, what patients can expect to be told and what rights they have to make the final decision. It also explains that whilst the views of people close to patients who lack capacity to make decisions are important, they cannot always be followed, especially where they conflict with the patients' interests or there is disagreement amongst family members. As with the guidelines for health professionals, the aim is to provide a framework to be adapted for local use.

The full text of the guidelines for health professionals and the views of three commentators follow. Footnotes have been omitted from the text of the guidelines but may be found at www.bma.org.uk/cpr

Gillian Romano-Critchley is Senior Ethics Advisor in the BMA's Medical Ethics Department. Ann Sommerville is Head of Ethics in the same department. 